\title{
Preparation and In Vitro Release of Ramose Chitosan-Based-5-Fluorouracil Microspheres
}

\author{
He-ping Li*, Hui Li, Zhou-dong Wang, Juan-juan Zhang, Man-feng Deng, and San-long Chen \\ School of Chemistry and Biologic Engineering, Changsha University of Science and Technology, \\ Changsha 410004, China. "E-mail: lihp@csust.edu.cn \\ (Received October 2, 2012; Accepted November 30, 2012)
}

\begin{abstract}
In order to construct a controlled release system of drugs and to reduce toxic side effects of 5-fluorouracil, the novel ramose chitosan-based-5-fluorouracil microspheres (CS-FU-MS) were prepared. Firstly, using chitosan (CS) as carriers and 5-fluorouracil (5-FU) as a model drug, ramose chitosan-based-5-fluorouracil (CS-FU) was efciently synthesized by chemical crosslinking method through microwave irradiation, drug loading was $10.6 \%$; Secondly, CS-FU-MS were prepared by CS-FU self-assembled under the dialysis conditions and the free 5-FU was encapsulated further at the same time. The size dispersivity of particles is uniform, and the average diameter of the CS-FU-MS was $4 \mu \mathrm{m}$. The drug encapsulation efficiency was $76.1 \%$, and the drug loading was increased to $26.22 \%$. CS-FU-MS maintain the zero-order release time in PBS (pH $=7.4)$ and $\mathrm{HCl} / \mathrm{KCl}(\mathrm{pH}=1.2)$ dialysis medium was $40 \mathrm{~h}$ and $34 \mathrm{~h}$ respectively, and the cumulative release were $58.89 \%$ and $79.33 \%$ in $182 \mathrm{~h}$. The results showed that CS-FU-MS have excellent sustained release properties.
\end{abstract}

Key words: Chitosan-based-5-fluorouracil, Preparation, Controlled release, Microspheres

\section{INTRODUCTION}

5-fluorouracil (5-FU) has been used as a kind of potent antitumor drug in clinical practice since the late $1950 \mathrm{~s} .{ }^{1}$ Unfortunately, 5-FU exhibited serious toxicity and aroused many side effects, such as begetting bone marrow depression, nausea, leucopenia, thrombocytopenia, or even heart toxicosis symptom. ${ }^{2}$ In addition, it has a short plasma halflife period of 10-20 $\mathrm{min}$ in vivo, low bioavailability, ${ }^{3}$ and should be administrated duratively by intravenous injection in clinical application. In order to prolong its half-life period and then reinforce antitumor curative effects as well as mitigate the side effects, a lot of 5-FU derivatives were synthesized by chemical modification. ${ }^{4}$ These derivates are able to overcome the over-mentioned shortcomings. However, their release processes still cannot be controlled. Therefore, using organic polymer as drug carrier is an important research field in recent years. ${ }^{5}$

Chitosan (CS) has been widely used in drug carriers for its biodegradability, biocompatibility and nontoxic properties. ${ }^{6}$ It can also induce the production of interferon and tumor necrosis factor, improve immunity and tumor cells apoptosis through activating immune cells in vivo. ${ }^{7}$ Consequently, CS plays the role of adjuvant therapy. Usually, drugs are loaded to chitosan by either chemical linking or microspheres embedding. ${ }^{8}$ The chemical linking system has the shortcomings of lower drug loading and too slower releasing rate. The embedding system has the disadvantages of weaker mechanical strength of controlled-release capsule or membrane that its rupture would lead to unsatisfactory release. In order to avoid the defects of simplex method, chitosan-based-5-fluorouracil (CS-FU) was synthesized firstly, 5-FU could evenly distribution in the carrier interior through chemical binding, and with the slow degradation of the relevant chemical bonds, the drugs can be slowly released. Then, chitosan-based-5-fluorouracial microspheres (CS-FU-MS) were prepared by using CSFU as capsules for encapsulating free 5-FU, more 5-FU was loaded in the mocrospheres, and the drug loading was enlarged. CS-FU-MS are the combination of both chemical linking and microspheres embedding, which makes the system has more ideal properties.

\section{EXPERIMENTAL}

\section{Materials and Instruments}

CS was purchased from Zhejiang Yuhuan Ocean Biochemistry Co, Ltd.(Zhejiang, China), underwent further deacetylation through microwave irradiation in our laboratory so as to gain a deacetylation degree of $98.87 \%$ as determined by acid-base titration and viscosity-average molecular weight of $411 \mathrm{kDa}(\mathrm{MW}=411 \mathrm{kDa})$. 3-Bromopropanoic acid, N-hydroxysuccinimide (NHS) and 1(3-(dimethylamino)-propyl)-3-ethylcarbodiimide hydro- 
chloride (EDC) were purchased from Sinopharm Chemical Reagent Co, Ltd. (Shanghai, China); 5-FU (99\%) was purchased from J\&K Chemical Ltd. (Beijing, China); 1,3-bis (hydroxymethyl)-5-fluorouracil (HMFU) was synthesized by our lab; All other reagents were analytical grade and used without further purification. The dialysis tubes ( $\mathrm{Mw}$ cut-off $8 \mathrm{kDa}$ ) were purchased from Ding States Biotechnology Limited Liability Company (Beijing, China).

Freeze Dryer (FD-1A type, Beijing Medical Kang Bo Experimental Instrument Co, Ltd, Beijing, China); Microwave oven (H6318-type, Guangdong, China); Infrared spectrometer (Avatar FT/IR-360, Nicolet, the United States, $\mathrm{KBr}$ tablet method); Laser particle size analyzer (winner 2000, Jinan Winner Instruments Co, Ltd.); Scanning electron microscope (JSM-6400F, Japan's Hitachi Ltd.); UV-Vis double beam spectrophotometer (TU-1901 type, Beijing general analysis of GM).

\section{Synthesis of N,N-dicarboxyethyl Chitosan (CECS)}

The synthesis of CECS was according to the literature. ${ }^{9}$ Briefly, CS $\left(0.4 \mathrm{~g}, 2.3 \mathrm{mmol}\right.$ of free $\left.-\mathrm{NH}_{2}\right)$ and 3-bromopropanoic acid $\left(5.3 \mathrm{~g}, 15\right.$ eq. $-\mathrm{NH}_{2}$ ) were dissolved in distilled water $(30 \mathrm{~mL})$, moderate amount of $\mathrm{NaHCO}_{3}$ was added to adjust $\mathrm{pH}$ value to 8.5 . The solution was intermittently radiated by microwave (xed power of $550 \mathrm{~W}$ ) at $60 \pm 3{ }^{\circ} \mathrm{C}$ for $50 \mathrm{~min}$, and $\mathrm{NaHCO}_{3}$ was added cautiously to keep $\mathrm{pH}$ value at 8.5. After cooled to 0 , the nal solution was poured into anhydrous alcohol $(40 \mathrm{~mL})$ and then light yellow precipitates were separated out. The precipitates were dissolved in water $(10 \mathrm{~mL})$, acidied to $\mathrm{pH} 1-2$ with concentrated $\mathrm{HCl}$, dialyzed against deionized water for 3 days, and freeze-dried to get CECS. IR(KBr): the strong absorption peak was at $1715 \mathrm{~cm}^{-1}(\mathrm{C}=\mathrm{O})$.

\section{Synthesis of Chitosan-based-5-uorouracil (CS-FU)}

The synthesis of CS-FU was according to the literature. ${ }^{9}$ Briefly, CECS $(0.0500 \mathrm{~g})$ was dissolved in distilled water $(2 \mathrm{~mL})$ followed by adding EDC $(0.3834 \mathrm{~g}, 2 \mathrm{mmol})$ and NHS ( $0.0460 \mathrm{~g}, 0.4 \mathrm{mmol})$, and then the mixture was activated with ultrasonic for $1 \mathrm{~h}$ at 0, After that, $\operatorname{HMFU}(0.1000 \mathrm{~g})$ was added, and the mixture was stirred for $24 \mathrm{~h}$ at $25^{\circ} \mathrm{C}$. The solution was dialyzed against deionized water in the dialysis tube for 3 days and freeze-dried to get CS-FU. IR (KBr): the strong absorption band was at $3081 \mathrm{~cm}^{-1}$ (the $\mathrm{C}-\mathrm{H}$ stretching connected with the $\mathrm{C}=\mathrm{C}$ contained in 5-FU).

\section{Preparation of Chitosan-based-5-fluorouracil Micro- spheres (CS-FU-MS) \\ CS-FU (20 mg) and moderate amount of 5-FU were dis-}

solved in $\mathrm{HCl}\left(10 \mathrm{~mL}, 1 \mathrm{~mol} \cdot \mathrm{L}^{-1}\right)$ heat for $5 \mathrm{~min}$ at $70^{\circ} \mathrm{C}$, then the solution was dialyzed with deionized water in the dialysis tube, until the liquid of dialysis tube appeared opalescence phenomenon firstly, high-speed centrifugation and then removed the supernatant, the residue product was freeze-dried finally.

\section{The DeterminatIon Of Drug Loading and Encapsu- lation Efficiency}

Series of standard solution of 5-FU were prepared, which absorbency was measured at $265 \mathrm{~nm}$. Standard curve was plotted according to the absorbency, and equation was obtained in linear regression as follows:

$$
\mathrm{Abs}=0.36646 \mathrm{C} / \mathrm{mg} \cdot \mathrm{L}^{-1}+0.00331(\mathrm{R}=0.9993)
$$

Absorbency of CS-FU (having unknown drug loading) solution was measured at $265 \mathrm{~nm}$. The data were embedded in Eq. 1 to obtain the content of 5-FU. The drug loading was calculated as follows: ${ }^{10}$

$$
\text { Drug loading }=\frac{\text { the content of } 5 \text {-FU in CS-FU }}{\text { the amount of CS-FU }} \times 100 \%
$$

CS-FU-MS (15 mg, freeze-dried) was dissolved in $10 \mathrm{~mL}$ water and measured absorbance at $265 \mathrm{~nm}$, and the data were embedded in Eq. 1 to obtain the content of 5-FU, the drug loading of CS-FU-MS was calculated as the same way of Eq. 2, and the encapsulation efficiency was calculated as follows:

$$
\begin{aligned}
& \text { Encapsulation efficiency }= \\
& \text { the content of 5-FU in CS-FU-MS - 5-FU in CS-FU } \\
& \times 100 \%
\end{aligned}
$$

\section{Particles Size Analysis and SEM}

Put CS-FU-MS suspension into ultrasonic for $5 \mathrm{~min}$, then the particles size and size distribution of microspheres were determined by a laser particle size analyzer, and the morphology was measured by scanning electron microscope (SEM) ${ }^{11}$ The dispersion of microspheres were added to the sample dispersion unit, and stirred in order to reduce the aggregation between the microspheres, the laser obscuration range was maintained between $3 \%$ and $15 \%$. The size distribution and mean particle size were measured after performing the experiment in triplicate. The shape and surface of the microspheres were evaluated by SEM. The samples were mounted directly onto the SEM sample holder using double-sided sticking tape and images were recorded at the required magnification at the acceleration voltage of $5 \mathrm{kV}$. 


\section{In Vitro Release}

CS-FU-MS (5 mg) was dispersed in $5 \mathrm{~mL}$ phosphate buffer solution (PBS, $\mathrm{pH}=7.4$ ) and placed in dialysis tube membrane, tied and placed into $100 \mathrm{~mL}$ PBS medium as above at $37^{\circ} \mathrm{C} \pm 0.5^{\circ} \mathrm{C}$ in a jar with stirring $(50 \mathrm{rpm} / \mathrm{min})$, $5 \mathrm{~mL}$ dialysis medium was taken out at each certain interval followed by replenishing the same volume of fresh medium. The concentration of 5-FU was obtained as mentioned above in Eq. 1. ${ }^{12}$ The in vitro release of CS-FU-MS in $\mathrm{HCl}$ / $\mathrm{KCl}$ solution $(\mathrm{pH}=1.2)$ was performed using the same way. The cumulative release was calculated as follows: ${ }^{13}$

$$
\text { Cumulative release }=\frac{V_{0} \times c_{t}+V \times \sum_{n=1}^{t-1} c}{W \times X} \times 100 \%
$$

where $V_{0}$ is the volume of PBS medium at the first $t$ time, $V$ is the volume of sampling PBS medium taken out at each certain interval, $c_{t}$ is the concentration of 5-FU in PBS medium at the first $\mathbf{t}$ time, $c$ is the concentration of 5FU in sampling PBS medium, $W$ is the amount of CS-FUMS, $X$ is the drug loading of CS-FU-MS.

\section{RESULTS AND DISSCUSIONS}

\section{Synthesis of CS-FU}

The synthetic route of CS-FU was showed in Scheme 1. Microwave irradiation has the advantages of significantly speeding up the reaction rate, increasing yield and reduc-
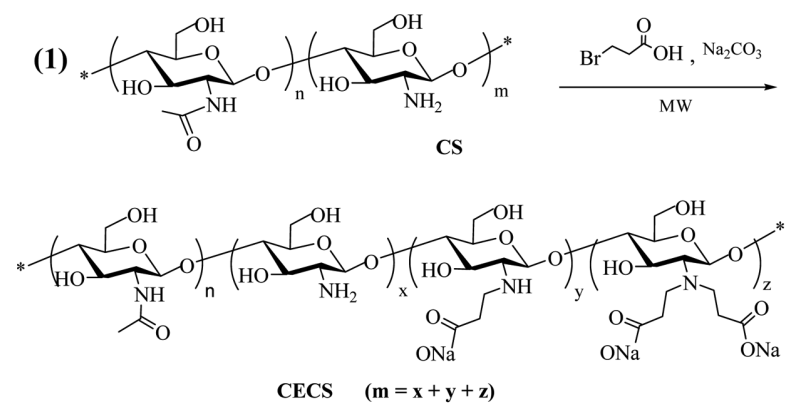

(2)
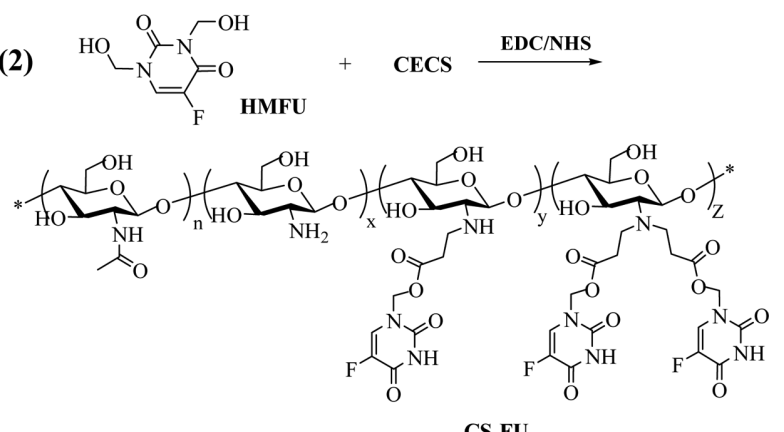

Scheme 1. Synthetic route of CS-FU. ing the adverse reactions and so on. In the synthetic process of CECS, compared to traditional heating way, microwave irradiation can significantly accelerate to 50 times. ${ }^{14}$ But in the synthetic process of CS-FU, the microwave irradiation can speed up the crosslinking reaction, bring on the gel formation. In this work, using the EDC / NHS as catalysts, CS-FU was synthesized successfully at room temperature. ${ }^{9}$ It was found that reaction time was the most important influence factor in this reaction. The influence of reaction time on the drug loading and gel formation were shown in Fig. 1 and Fig. 2 respectively.

It can be seen from the Fig. 1 that drug loading was increased with the reaction time prolonging, drug loading reached $10.60 \%$ after $24 \mathrm{~h}$, and only reach $10.67 \%$ after 28 h. From Fig. 2, it was seen that the formation of the gel was affected significantly by the reaction time, after $28 \mathrm{~h}$, the gel got to $61.54 \%$ of the total product and affected the yield seriously.

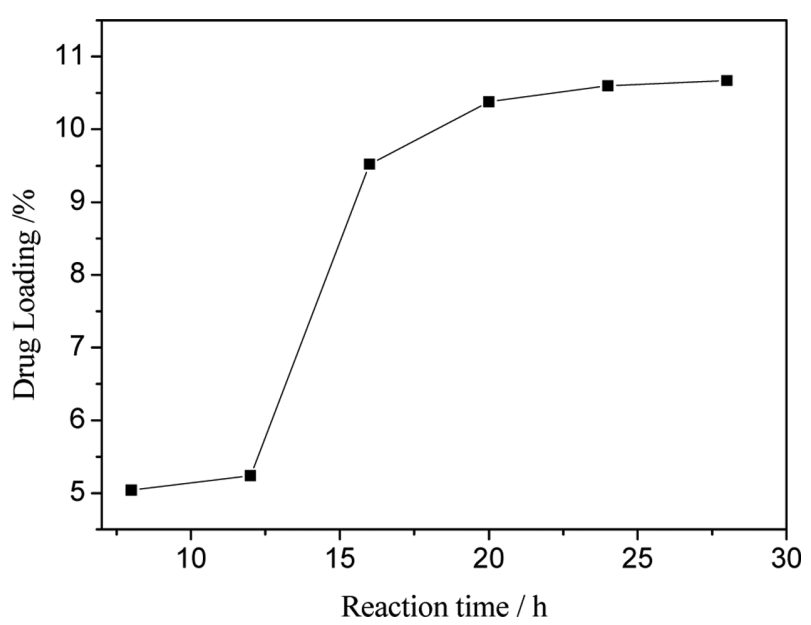

Fig. 1. The drug loading of CS-FU at different reaction time.

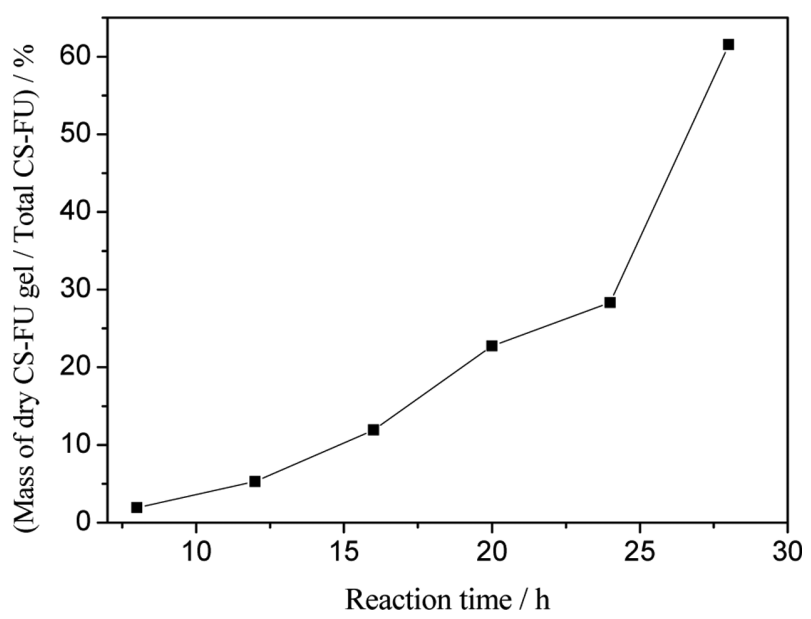

Fig. 2. The amount of gel at different reaction time. 


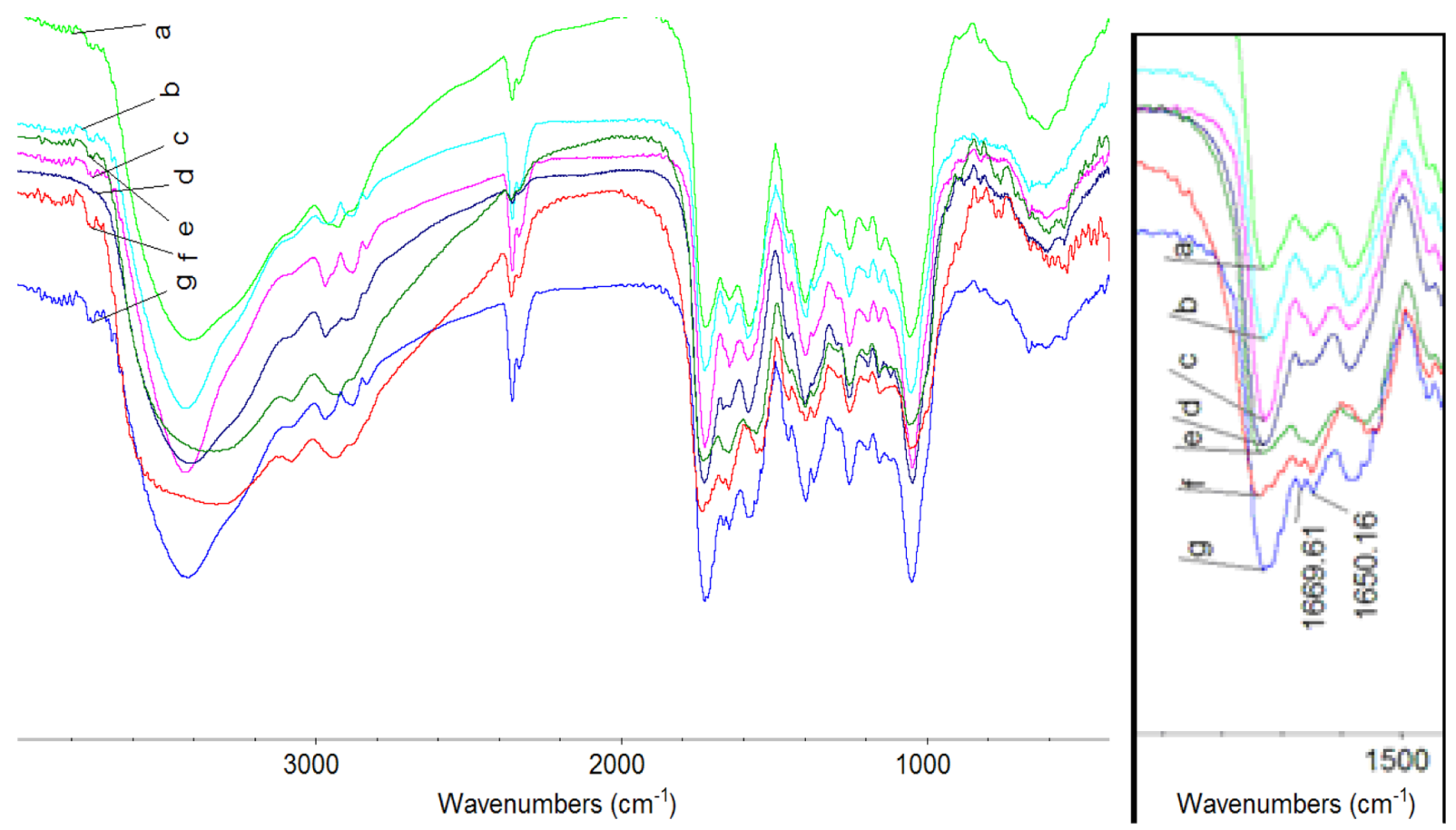

Fig. 3. FTIR spectra of CS-FU and gel (a: CS-FU of reaction $8 \mathrm{~h}$; b: CS-FU of reaction $12 \mathrm{~h}$; c: CS-FU of reaction $16 \mathrm{~h}$; d: CS-FU of reaction $20 \mathrm{~h}$; e: CS-FU of reaction $24 \mathrm{~h}$; f: gel of reaction $24 \mathrm{~h}$; g: gel of reaction 28h).

At different time the infrared spectrum of CS-FU and gel were presented in Fig. 3.

In the Fig. 3, the absorption peak at $1650 \mathrm{~cm}^{-1}$ is due to $\mathrm{C}=\mathrm{C}$ stretching vibration of 5-Fu ring; The peak at 1669 $\mathrm{cm}^{-1}$ is due to $\mathrm{C}=\mathrm{O}$ stretching vibration of crosslinking acylamide. From Fig. 3a to Fig. 3g, the peak at $1669 \mathrm{~cm}^{-1}$ is strengthening, which indicates that the reaction between $-\mathrm{NH}_{2}$ and $-\mathrm{COONa}$ was happened and crosslinking was increased. After $28 \mathrm{~h}$, the peak of acylamide was obvious, which indicates that the amount of gel was increased. Considering the factors of drug loading and gelating side reaction, the most suitable reaction time is $24 \mathrm{~h}$.

\section{Preparation of CS-FU-MS}

The preparation of CS-FU-MS can be concluded from the presence of opalescence phenomenon in dialysis. The particle size of CS-FU-MS was found to be dependent on the time of dialysis. In fact, the more time of dialysis was given, the more obvious the opalescence phenomenon appeared, and the larger diameter of CS-FU-MS was obtained. When the time of dialysis was excessively long, CS-FU would be converted to the shape of flocculation agglomerate. The drug loading and encapsulation efficiency of CS-FU-MS were $26.22 \%$ and $76.1 \%$ respectively. The particle size distribution pattern of the CS-FU-MS was shown in Fig. 4. The size distribution of CS-FU-MS was 95\% with

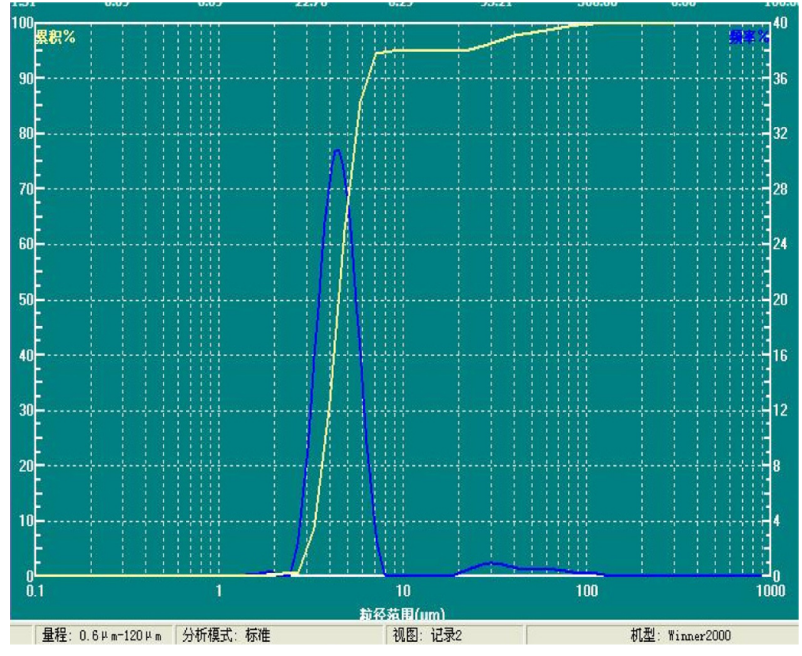

Fig. 4. Particle size distribution curve of CS-FU-MS.

the diameter of 3-6 $\mu \mathrm{m}$. The particle size dispersivity was uniform, and the average diameter of the CS-FU-MS was $4 \mu \mathrm{m}$. SEM images of the CS-FU-MS were shown in Fig. 5. SEM studies indicated that the surface of CS-FU-MS was smooth, without hollows or deformation, and the diameter was $3-5 \mu \mathrm{m}$.

In Vitro Release of $C S-F U-M S$

Release of 5-FU from CS-FU-MS involves three dif- 


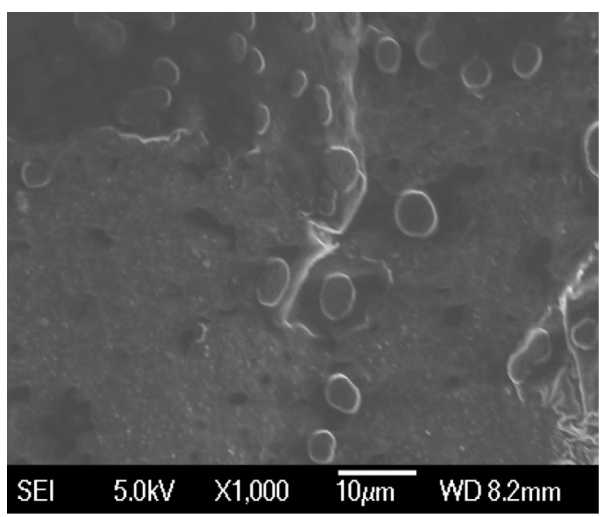

Fig. 5. SEM photograph of CS-FU-MS.

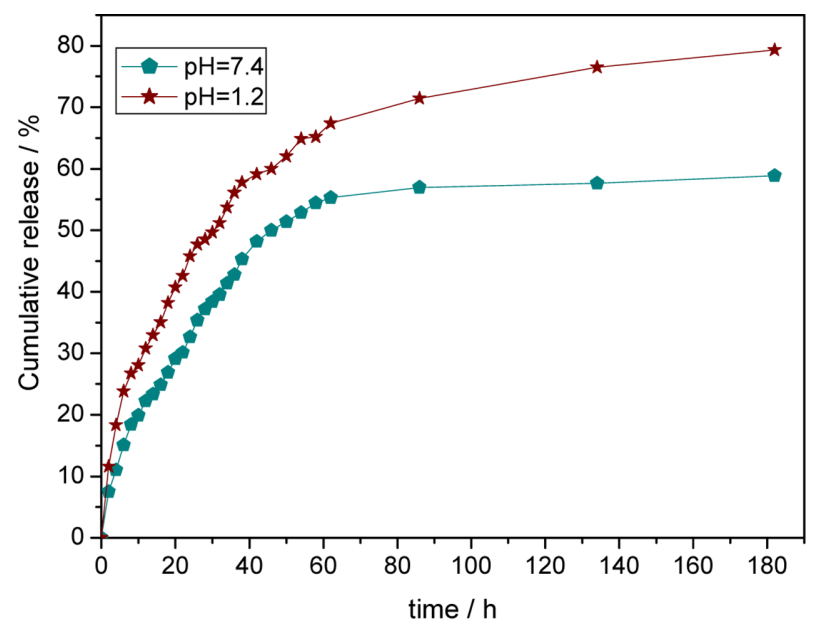

Fig. 6. Cumulative release of CS-FU-MS in PBS $(\mathrm{pH}=7.4)$ and $\mathrm{HCl} / \mathrm{KCl}(\mathrm{pH}=1.2)$ at $37 \pm 0.5^{\circ} \mathrm{C}$.

ferent mechanisms: (a) releasing from the surface of microspheres, (b) diffusion through the swollen rubbery matrix, and (c) releasing due to chemical bond hydrolysis. ${ }^{15}$ In order to simulate the medicine release performance of CSFU-MS in the small intestine and the gastric juice $\mathrm{pH}$ environment, the release behaviours and stages of 5-FU from CS-FU-MS in $\mathrm{PBS}(\mathrm{pH}=7.4)$ and $\mathrm{HCl} / \mathrm{KCl}$ solution $(\mathrm{pH}=1.2)$ were shown in Fig. 6.

The release of 5-FU was dependent on $\mathrm{pH}$ value of medium conditions, with slower release kinetics at higher $\mathrm{pH}$ conditions, which was attributed to $-\mathrm{NH}_{2}$ and $-\mathrm{OH}$ of chitosan protonation resulting in a soluble, and positively charged chitosan leading to faster swelling in acidic medium. The release data revealed that there were three stages for the release of 5-FU from CS-FU-MS. The first stage of release (within $6 \mathrm{~h}$ ) was initially rapid (burst release), which may be result from the rapid diffusion of 5-FU onto the surface of CS-FU-MS from the initial swelling of the spheres. The second stage of release $(6-60 \mathrm{~h})$ was slower than that of the first stage, which was attributing to the combination of diffusion and hydrolysis (controlled release). Later, the third stage of release $(60-180 \mathrm{~h}$ ) was very slow from CS-FU-MS (controlled release), which may be result from the slow hydrolysis of CS-FU molecules into the solution. The CS-FU-MS in PBS $(\mathrm{pH}=7.4)$ and $\mathrm{HCl} / \mathrm{KCl}$ $(\mathrm{pH}=1.2)$ dialysis medium could maintain the zero-order release time was $40 \mathrm{~h}$ and $34 \mathrm{~h}$ respectively, and the percentage of cumulative release were $58.89 \%$ and $79.33 \%$ in $182 \mathrm{~h}$. The burst release in first stage may help to reach the effective concentration of 5-FU rapidly in plasma, whereas the controlled release would maintain the effective concentration of 5-FU in plasma for a long time. The results suggested that the effective concentration of 5-FU could be supplemented by encapsulation methods, and thus prolonged its biological half-time in vivo.

\section{CONCLUSIONS}

(1) CS-FU-MS were prepared with the self-assembly method of dialysis, the average size of particles is $4 \mu \mathrm{m}$. CS-FU-MS was proved to have higher drug loading and encapsulation efficiency.

(2) CS-FU-MS exhibited excellent releasing effect to meet application in clinic.

Acknowledgment. This work was financially supported by the National Natural Science Foundation of China (No. 20775010), the Natural Science Foundation of Hunan Province of China (06JJ2038) and Graduate Innovation Fund of Hunan Province of China.

\section{REFERENCES}

1. Parker, J. B.; Stivers, J. T. Biochemistry 2011, 50(5), 612.

2. Francini, G.; Petrioli, R.; Aquino, A.; Gonnelli, S. Cancer. Chemother. Pharmacol 1993, 32, 359.

3. (a) Shuai, X. T.; Ai, H.; Nasongkl, N.; Kim, S.; Gao, J. M. J. Controlled. Release. 2004, 98, 415. (b) Yu, C. Y.; Jia, L. H.; Yin, B. C.; Zhang, X. Z.; Cheng, S. X.; Zhuo, R. X. J. Phys. Chem. 2008, 112, 16774.

4. Akalin, E.; Akyuz, S.; Akyuz, T. J. Mol. Struct. 2007, 834$836,477$.

5. (a) De Queiroz, A. A. A.; Abraham, G. A.; Higa, O.Z. Acta. Biomater. 2006, 2, 641. (b) Putnam, D.; Kopecek, J. Bioconjug. Chem. 1995, 6, 483.

6. (a) Luo, Y. C.; Zhang, B. C.; Whent, M.; Yu, L. L.; Wang, Q. Colloids Surf. B 2011, 85, 145. (b) Lee, E.; Lee, J.; Lee, I. H.; Yu, M.; Kim, H.; Chae, S. Y.; Jon, S. J. Med. Chem. 2008, 51, 6442.

7. (a) Maeda, Y.; Kimura, Y. J. Nutr. 2004, 134, 945. (b) 
Seferian, P. G.; Martinez, M. L. Vaccine 2000, 19, 661.

8. Weerakody, R.; Fagan, P.; Kosaraju, Shantha L. Int. J. Pharm. 2008, 357(1-2), 213.

9. Li, H. P.; Wang, Z. D.; Yu, T. Med. Chem. Res. 2011, 20, 67.

10. Zheng, Y. L.; Yang, W. L.; Wang C. C.; Hu J. H.; Fu, S. K.; Dong, L.; Wu, L. L.; Shen, X. Z. Eur. J. Pharm. Biopharm. 2007, 67, 6211.

11. Batista, M. K. S.; Pinto, L. F.; Gomes, C. A. R.; Gomes, P. Carbohydr. Polym. 2006, 64, 299.
12. Pierre, D.; Caroline, D.; Jean-Michel, Chezal.; Eric, D.; Michel, M.; Pascal, C.; Marie-Josèphe, G. Eur. J. Med. Chem. 2011, 46, 2867.

13. Chen, G. G.; Zhou, B. Q.; Li, X. M.; Wei, P. J. Chin. Pharm. Univ. 2006, 37, 423.

14. Ge, H. C.; Pang, W.; Luo, D. K. Carbohydr. Polym. 2006, 66, 372

15. Peng, H. L.; Xiong, H.; Li, J. H.; Xie, M. Y.; Liu, Y. Z.; Bai, C. Q.; Chen, L. X. Food Chem. 2010, 121, 23. 